\title{
Improving hospital-based end of life care processes and outcomes: a systematic review of research output, quality and effectiveness
}

\author{
Amy Waller ${ }^{1,2^{*}}$, Natalie Dodd ${ }^{1,2}$, Martin H. N. Tattersall ${ }^{3}$, Balakrishnan Nair ${ }^{4,5}$ and Rob Sanson-Fisher ${ }^{1,2}$
}

\begin{abstract}
Background: As in other areas of health delivery, there is a need to ensure that end-of-life care is guided by patient centred research. A systematic review was undertaken to examine the quantity and quality of data-based research aimed at improving the (a) processes and (b) outcomes associated with delivering end-of-life care in hospital settings.

Methods: Medline, EMBASE and Cochrane databases were searched between 1995 and 2015 for data-based papers. Eligible papers were classified as descriptive, measurement or intervention studies. Intervention studies were categorised according to whether the primary aim was to improve: (a) end of life processes (i.e. end-of-life documentation and discussions, referrals); or (b) end-of-life outcomes (i.e. perceived quality of life, health status, health care use, costs). Intervention studies were assessed against the Effective Practice and Organisation of Care methodological criteria for research design, and their effectiveness examined.

Results: A total of 416 papers met eligibility criteria. The number increased by $13 \%$ each year $(p<0.001)$. Most studies were descriptive $(n=351,85 \%)$, with fewer measurement $(n=17)$ and intervention studies $(n=48 ; 10 \%)$. Only 18 intervention studies (4\%) met EPOC design criteria. Most reported benefits for end-of-life processes including end-of-life discussions and documentation (9/11). Impact on end-of-life outcomes was mixed, with some benefit for psychosocial distress, satisfaction and concordance in care (3/7).

Conclusion: More methodologically robust studies are needed to evaluate the impact of interventions on end-of-life processes, including whether changes in processes translate to improved end-of-life outcomes. Interventions which target both the patient and substitute decision maker in an effort to achieve these changes would be beneficial.
\end{abstract}

Keywords: End-of-life, Hospital, Advance care planning, Palliative care, Acute care

\section{Background}

Between one-third and two thirds of people in developed world countries will die in hospital, and approximately $20 \%$ of people will die in an intensive care unit $[1,2]$. People living longer with chronic diseases and limited availability and access to well-resourced community services have contributed to the increasing trend for

\footnotetext{
* Correspondence: amy.waller@newcastle.edu.au

${ }^{1}$ Priority Research Centre in Health Behaviour, University of Newcastle, Callaghan, NSW 2308, Australia

${ }^{2}$ Hunter Medical Research Institute, Newcastle, NSW 2305, Australia Full list of author information is available at the end of the article
}

institution-based deaths [3, 4]. However, many people either do not understand or are unaware of end-of-life care options [5]. Health care providers involved in the care of dying patients report difficulties in knowing when and how to withdraw or withhold life-sustaining treatments $[5,6]$. Dying in hospital has been associated with high rates of unwanted aggressive treatment, underuse or late use of palliative care and poorer symptom management [7-10].

Process and consequences of health delivery are important aspects of care to measure. Processes include those things that are in immediate control of healthcare 
providers and are intended to improve the outcomes associated with end-of-life care, such as goals of care discussions; end-of-life documentation (e.g. advance care directives (ACDs), do-not-resuscitate (DNR) orders); involvement of support persons in decision-making; and referrals to hospice. End-of-life outcomes are seen as functions of the processes of care patients undergo and the structures in which these processes occur (e.g. hospital, skill mix). End-of-life outcomes may include perceived health status, quality of life; concordance between preferred and actual care; survival; and costs or utilization. It is expected that successful implementation of end-of-life processes will be associated with improved end-of-life outcomes.

A number of approaches are hypothesised as a means of improving end-of-life processes and outcomes, including advance care planning, family meetings and palliative care consultations. Previous systematic reviews have synthesised the literature across a range of care settings for certain interventions, such as ACP $[11,12]$. Others have focused on the impact of different interventions in care settings, such as the intensive care unit (ICU) [13, 14]. There has been limited synthesis of the evidence pertaining to the impact of these interventions on end-of-life processes and outcomes in general hospital settings. This is an important gap for a number of reasons. First, the rise in the number of deaths occurring in this setting in many countries is likely to place increasing pressure on already finite resources, which may result in suboptimal care [3, 15]. Second, there are high personal and societal costs associated with suboptimal end-of-life care, highlighting the need for improvements. Third, the success of interventions may be dependent on the environmental context in which they are applied [16]. Interventions successfully applied to stable outpatients or in the general community may not achieve similar improvements in hospital.

Given the limited health service resources available, it is important that end of life care is evidence-based, rather than based solely on the intuition of service providers. Research must meet minimum standards of scientific quality to ensure adequate internal and external validity. [17]. Measurement studies involve the development of psychometric tools that can reliably and accurately assess end-oflife processes or outcomes. Such tools are used to provide empirical data describing the prevalence and correlates of the outcomes and inform how we might intervene to address important gaps in care. Intervention studies can provide evidence of effective strategies that can be implemented to reduce gaps. The quality of the studies must also be established; as high volume doesn't necessarily equate with quality. Despite potentially adverse consequences of suboptimal hospital-based end-of-life care, the quality, relevance and impact of research associated with end-of-life processes and outcomes in hospitals has not been examined.

\section{Methods}

Aims: This systematic review aimed to examine the:

1) volume and type of data-based publications examining end-of-life care among people dying in hospital and their families;

2) methodological quality of intervention studies aimed at improving end-of-life processes and outcomes according to EPOC methodological criteria; and

3) the effectiveness of interventions in studies that met this criteria.

\section{Search strategy}

A search of MEDLINE, EMBASE, and CINAHL databases was conducted by one author (AW) and a medical librarian independently (see acknowledgements) based on the search strategy in Fig. 1, limited to articles published between 1995 and December 2015. The search strategy for each of the databases is outlined in Additional file 1. Searches were restricted to human studies published in English.

\section{Inclusion and exclusion criteria}

Studies were included if they focused primarily on:

(a) end-of-life processes: end-of-life/goals of care discussions; end-of-life documentation (e.g. ACDs, DNR orders); appointment of substitute decision makers; medication orders; or referrals to hospice/ palliative care; and/or

(b)end-of-life care outcomes: health status, satisfaction and quality of life; perceived quality of care; concordance of preferred and actual care; survival; or health care costs or utilization;

(c) Studies examined these outcomes among adults (18 years or over) admitted to hospitals (excluding intensive care units) or their families.

Studies were excluded if they were book chapters, review articles, case studies, commentaries, conference abstracts, editorials or protocol papers.

\section{Data coding}

Paper titles were initially assessed against the eligibility criteria by AW and excluded if the study did not meet inclusion criteria. A random sub-sample (20\%) of included and excluded studies were categorised by another author (ND), with any discrepancies resolved via discussion. Papers were then categorized as either:

Measurement studies included those describing the development or testing of the psychometric quality of tools to assess either end-of-life care processes or outcomes. 


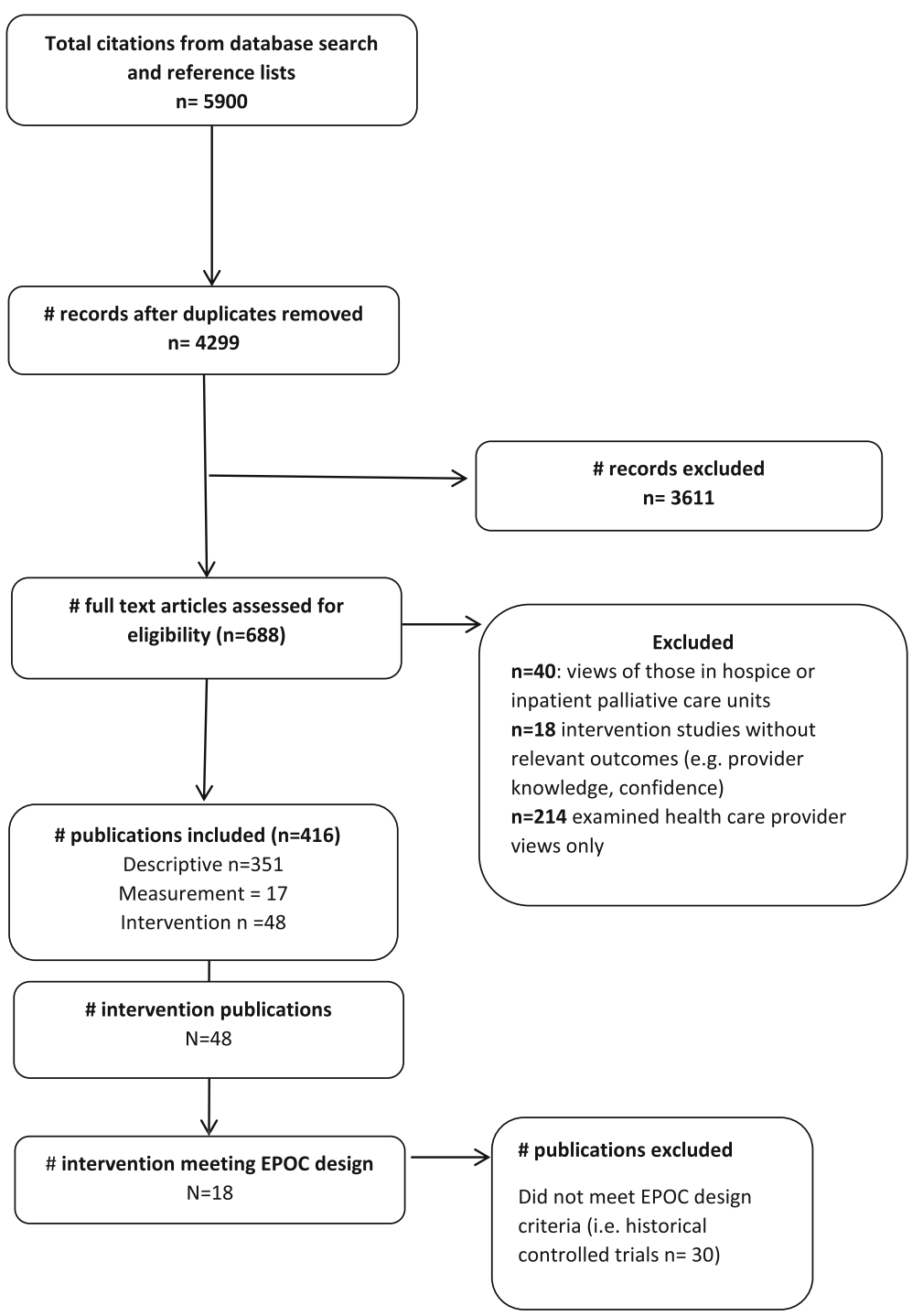

Fig. 1 Search strategy

Descriptive studies documented frequency, patterns, correlates and/or preferences in relation to either endof-life care processes or outcomes using quantitative or qualitative methods.

Intervention studies were categorised into two group: (1) those where the primary aim was to examine the impact of interventions on end-of-life processes; or (2) those where the primary aim was to examine the impact of the intervention on end-of-life outcomes.

\section{Assessment of methodological quality}

Intervention studies were assessed as to determine whether the experimental design was one of the four types allowed by the EPOC design criteria - randomized controlled trials, controlled trials, controlled before and after studies, or interrupted time series studies [18] Stepped wedge designs were also included as they are a viable alternative to a parallel cluster randomised trial and accepted by EPOC as a robust design. For those studies meeting minimum design criteria, methodological quality was then assessed using EPOC risk of bias criteria independently by two reviewers (AW and ND).

\section{Assessment of effectiveness}

Additional study data was extracted from each intervention study that met the minimum criteria for quality, including: aim; study setting; sample characteristics; inclusion and exclusion criteria; intervention design; outcome measures; follow-up periods and study findings. 


\section{Analysis}

Poisson regression was used to model trends over time in the numbers of publications. Percent change by year with Wald 95\% confidence are presented. $P$-values were calculated from the Wald Chi-square.

\section{Results}

\section{Search results}

A total of 4611 were identified for potential inclusion, after removal of duplicates. After assessment against eligibility criteria, 416 publications met criteria for inclusion in the review. A flow chart of the literature search and paper identification is provided in Fig. 1.

\section{Number and type of published studies of end-of-life care in hospitals (1995-2015)}

Poisson regression shows the number of publications increasing by $13 \%$ each year $(95 \% \mathrm{CI}=11.1-11.5 \%$; $P<0.0001$ ) (see Fig. 2). The majority of eligible studies were descriptive studies $(n=351,85 \%)$. Of these, 145 were descriptive studies describing the views of patients or carers $(n=145)$; and 206 were medical record audits. There were 17 measurement studies; with the remaining 48 studies reporting on interventions. Only 18 studies met EPOC design criteria (Table 1). Of these, 11 focused on end-of-life processes as their primary outcome [19-29]; and seven focused on end-of-life outcomes as their primary outcome [30-36] (Additional file 2).

\section{Methodological quality of studies}

Studies included a cluster randomised controlled trial [31]; a stepped wedge trial [19]; randomised control trials $[17,20-22,24,25,27,29,30,32-36]$; controlled clinical trials [20, 23] and an interrupted time series trials [26] (Table 1). Five studies were rated as low risk on at least seven of the nine criteria. The most poorly met criteria included: not specifying whether outcomes were assessed blindly or protected against contamination. Studies did not report on either method of generating allocation sequence or concealing allocation.

\section{Effectiveness of intervention studies meeting EPOC design criteria}

Table 2 presents the study characteristics of 18 intervention studies which were rated as high quality when compared to the EPOC criteria. Almost all of these studies were conducted in the USA [19-26, 28-30, 32, 34, 35], with one in the UK [27], one in Italy [31] and one in Australia [36]. Half of the studies targeted mixed seriously ill populations $[19,28,29,32,33]$. The remainder targeted the elderly [24, 36], surgical [22, 35], dementia [27, 29], heart failure [34]. Others included any admitted patient $[20,23,26]$. Three studies tested patient-directed interventions involving the provision of written information or audio-visual information [20, 21, 25]. Seven involved facilitated ACP interventions [22, 24, 27, 28, 30, 35, 36] and four were palliative care consultations [32-34, 29]. Four studies tested multi-faceted, system-based interventions [19, 23, 26, 31].

\section{Effectiveness of interventions examining end of life processes}

Two of three studies reported benefits for providing written or audio-visual information to hospitalized patients on completion of ACDs and CPR orders. Patient who received scripted information about cardiopulmonary resuscitation (CPR), mechanical ventilation, and ACDs more likely to clarify preferences for treatment and create ACDs [25]. A 3-min video about CPR and intubation improved documentation of CPR orders and intubation, patient knowledge and fewer seriously ill people chose these treatments compared to control patients [21]. However, videotaped interviews and written instructions did not improve ACD rates [20].

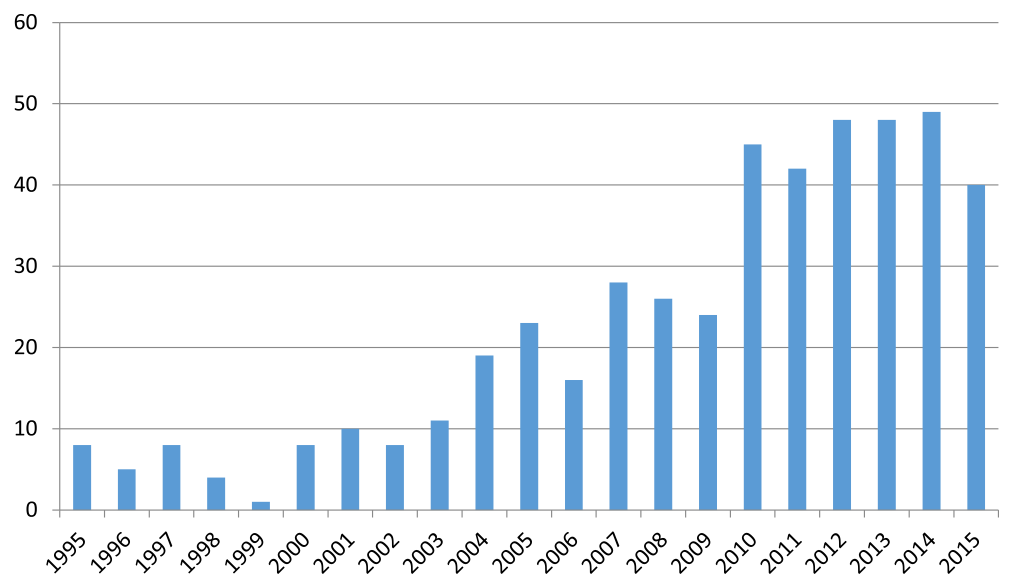

Fig. 2 Number of publications by year 
Table 1 Quality of intervention studies meeting EPOC design criteria (Low, High, Unclear)

\begin{tabular}{|c|c|c|c|c|c|c|c|c|c|c|}
\hline $\begin{array}{l}\text { Author, } \\
\text { Date Design }\end{array}$ & Type & $\begin{array}{l}\text { Allocation } \\
\text { sequence } \\
\text { adequately } \\
\text { generated? }\end{array}$ & $\begin{array}{l}\text { Concealment } \\
\text { of allocation }\end{array}$ & $\begin{array}{l}\text { Baseline } \\
\text { outcome } \\
\text { measurement } \\
\text { similar }\end{array}$ & $\begin{array}{l}\text { Baseline } \\
\text { characteristics } \\
\text { similar }\end{array}$ & $\begin{array}{l}\text { Incomplete } \\
\text { outcome } \\
\text { data } \\
\text { adequately } \\
\text { addressed }\end{array}$ & $\begin{array}{l}\text { Knowledge } \\
\text { of allocated } \\
\text { interventions } \\
\text { prevented }\end{array}$ & $\begin{array}{l}\text { Protections } \\
\text { against } \\
\text { contamination }\end{array}$ & $\begin{array}{l}\text { Selective } \\
\text { outcome } \\
\text { reporting }\end{array}$ & $\begin{array}{l}\text { Free } \\
\text { other } \\
\text { risk of } \\
\text { bias }\end{array}$ \\
\hline \multicolumn{11}{|c|}{ End of life outcomes } \\
\hline $\begin{array}{l}\text { Costantini } \\
2014[31]\end{array}$ & CRCT & L & L & L & L & L & $\mathrm{H}$ & L & L & $\mathrm{H}$ \\
\hline $\begin{array}{l}\text { Detering } \\
2010[36]\end{array}$ & RCT & L & L & L & L & L & L & $\mathrm{H}$ & L & L \\
\hline $\begin{array}{l}\text { Gade } 2008 \\
\text { [32] }\end{array}$ & RCT & L & L & L & $\mathrm{H}$ & $L$ & U & U & L & L \\
\hline $\begin{array}{l}\text { Hanks } 2002 \\
{[33]}\end{array}$ & $\mathrm{RCT}$ & L & L & L & $\mathrm{H}$ & L & $U$ & $U$ & L & L \\
\hline $\begin{array}{l}\text { Sidebottom } \\
2015 \text { [34] }\end{array}$ & RCT & $U$ & $U$ & L & $\mathrm{H}$ & L & U & $\mathrm{H}$ & L & L \\
\hline $\begin{array}{l}\text { Song } 2005 \\
{[35]}\end{array}$ & $\mathrm{RCT}$ & $U$ & U & L & L & $L$ & U & $U$ & L & L \\
\hline $\begin{array}{l}\text { The SUPPORT } \\
\text { Principal } \\
\text { Investigators } \\
1995 \text { [30] }\end{array}$ & $\mathrm{RCT}$ & L & L & L & L & $L$ & L & $U$ & L & L \\
\hline \multicolumn{11}{|c|}{ End of life processes } \\
\hline $\begin{array}{l}\text { Ahronheim } \\
2000 \text { [29] }\end{array}$ & $\mathrm{RCT}$ & $U$ & U & L & L & $L$ & L & $\mathrm{H}$ & L & $\mathrm{H}$ \\
\hline Bailey 2014 [19] & SW & $\mathrm{H}$ & L & L & L & L & L & L & L & $L$ \\
\hline $\begin{array}{l}\text { Cugliari } \\
1995[20]\end{array}$ & $\mathrm{CCT}$ & $\mathrm{H}$ & $\mathrm{H}$ & $U$ & $\mathrm{H}$ & $U$ & $U$ & $\mathrm{H}$ & L & L \\
\hline $\begin{array}{l}\text { El-Jawahri } \\
2015[21]\end{array}$ & RCT & L & L & L & L & $L$ & $\mathrm{H}$ & $U$ & L & L \\
\hline $\begin{array}{l}\text { Grimaldo } \\
2001[22]\end{array}$ & RCT & L & L & L & L & L & U & U & L & L \\
\hline $\begin{array}{l}\text { Jacobsen } \\
2011[23]\end{array}$ & $\mathrm{CCT}$ & $\mathrm{H}$ & $\mathrm{H}$ & $U$ & L & $L$ & $\mathrm{H}$ & L & L & L \\
\hline Meier 1996 [24] & $\mathrm{RCT}$ & $U$ & U & $U$ & L & L & $U$ & $U$ & L & L \\
\hline $\begin{array}{l}\text { Nicolasora } \\
2006[25]\end{array}$ & RCT & L & $U$ & $U$ & L & L & $\mathrm{H}$ & U & L & L \\
\hline $\begin{array}{l}\text { Sampson } \\
2011[27]\end{array}$ & RCT & U & U & L & L & $L$ & U & L & L & L \\
\hline Study (ITS) & \multicolumn{2}{|c|}{$\begin{array}{l}\text { Independent of } \\
\text { other changes? }\end{array}$} & $\begin{array}{l}\text { Shape of } \\
\text { effect pre- } \\
\text { specified? }\end{array}$ & $\begin{array}{l}\text { Intervention } \\
\text { affected data } \\
\text { collection? }\end{array}$ & $\begin{array}{l}\text { Knowledge } \\
\text { interventions } \\
\text { adequately } \\
\text { prevented }\end{array}$ & \multicolumn{2}{|c|}{$\begin{array}{l}\text { Incomplete outcome } \\
\text { data adequately addressed? }\end{array}$} & $\begin{array}{l}\text { Free from } \\
\text { selective } \\
\text { outcome } \\
\text { reporting? }\end{array}$ & \multicolumn{2}{|c|}{$\begin{array}{l}\text { Other risk of } \\
\text { bias? }\end{array}$} \\
\hline $\begin{array}{l}\text { Reilly } \\
1995 \text { [26] }\end{array}$ & L & & L & L & L & $L$ & & L & U & \\
\hline
\end{tabular}

More intensive strategies have had mixed success. Smaller studies of provider facilitated advance care planning interventions also reported benefits in surgical [22, 35 ] and elderly hospitalised patients [24]. Palliative care consultations were found to increase engagement in advance care planning among heart failure and mixed hospitalised populations [32, 34]. A multi-component system-based approach of site visits; a decision support tool; and staff education and training improved the rate of completion of ACDs and some, but not all medical orders [19]. In an ITS trial, completion of ACDs increased significantly during the intervention phase, as did agreement between ACDs and patient preferences [26]. Staff education, dedicated discussion time and increased palliative care involvement increased the rate of documented GOCD and limiting treatment orders [23]. 


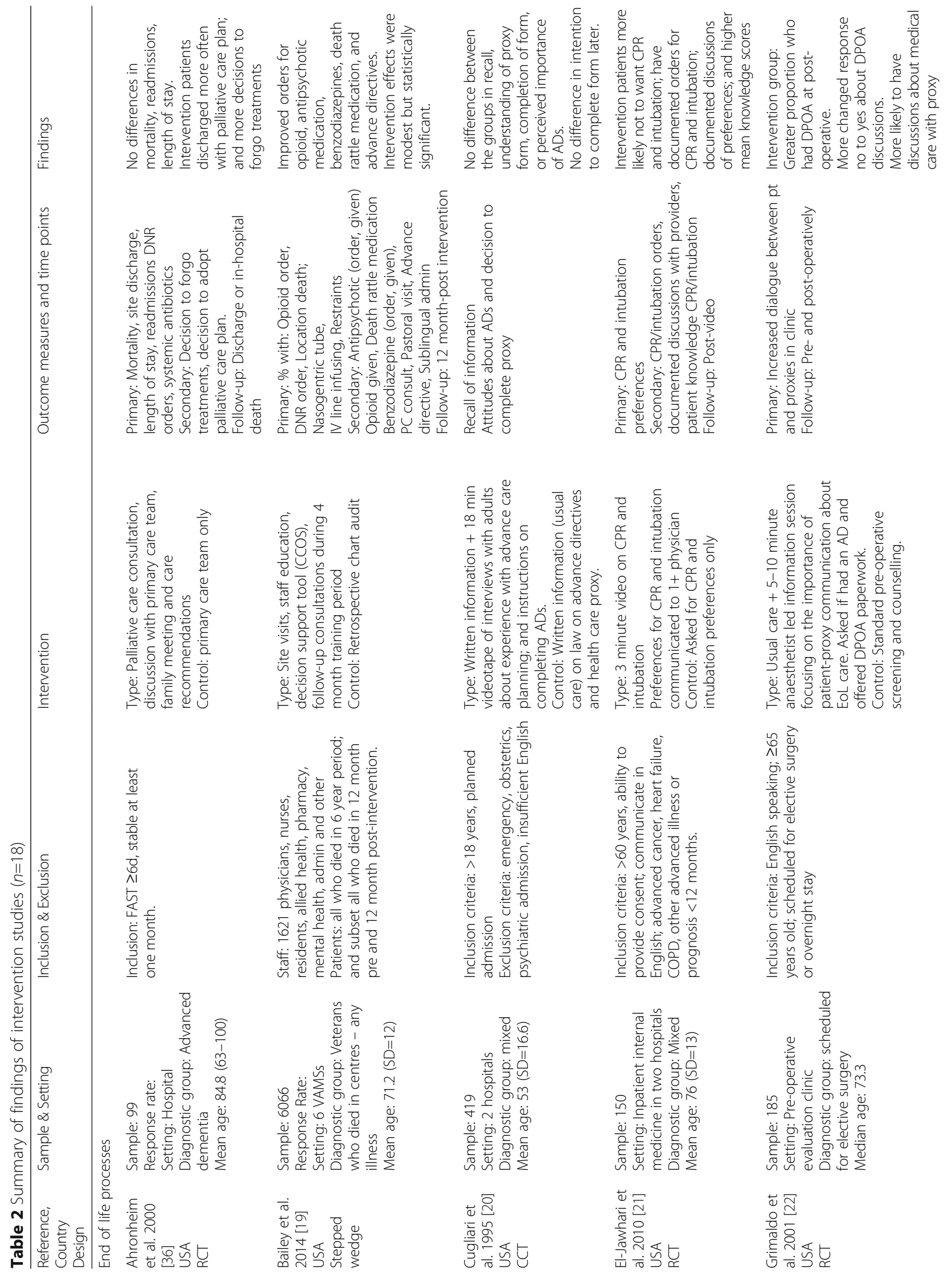




\begin{tabular}{|c|c|c|c|c|c|}
\hline 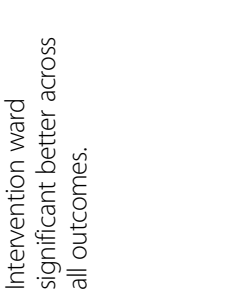 & 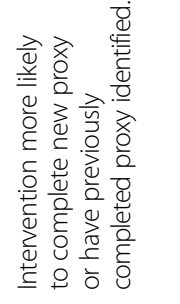 & 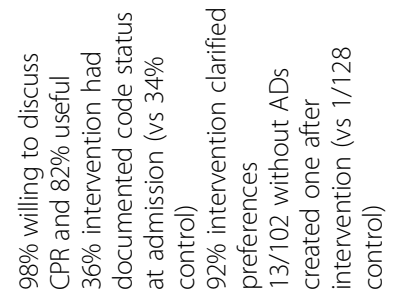 & 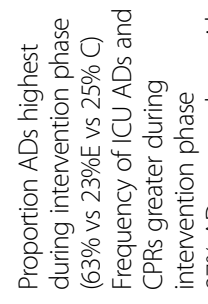 & 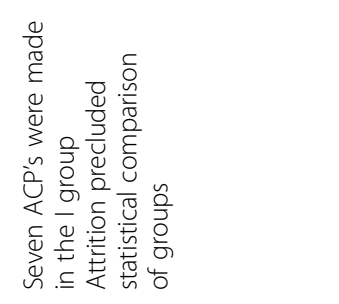 & 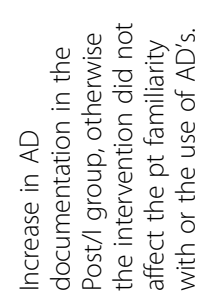 \\
\hline 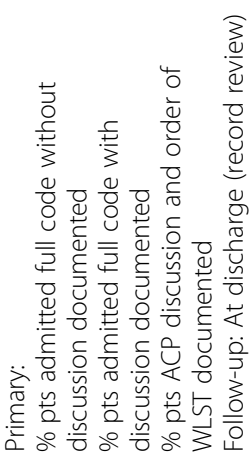 & 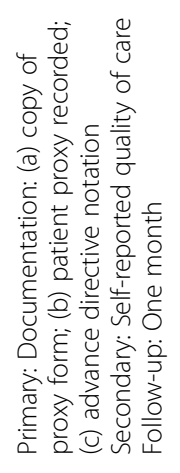 & 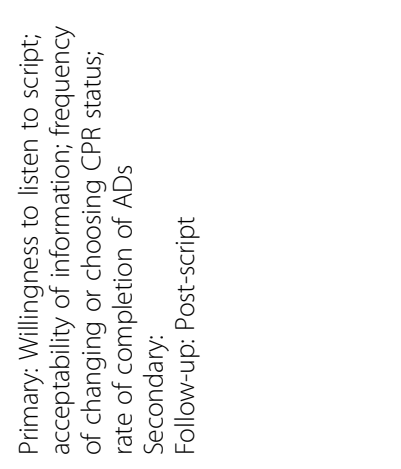 & 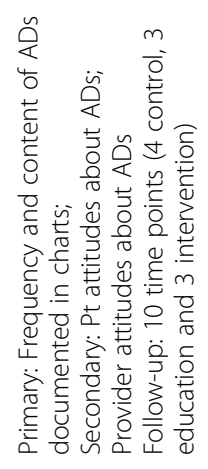 & 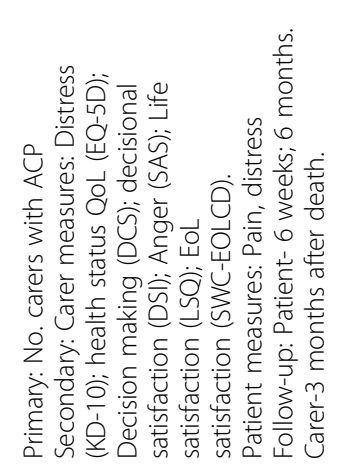 & 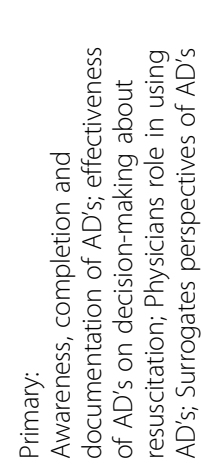 \\
\hline 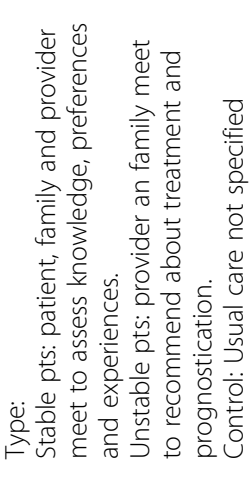 & 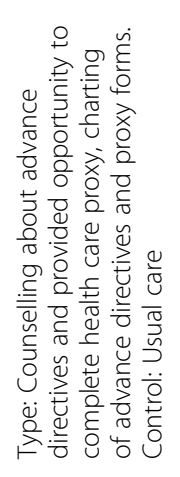 & 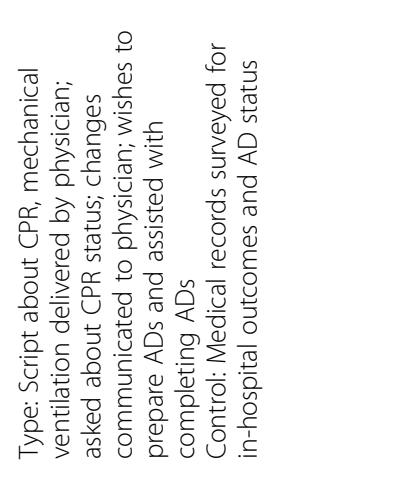 & 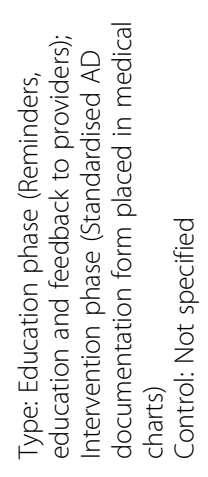 & 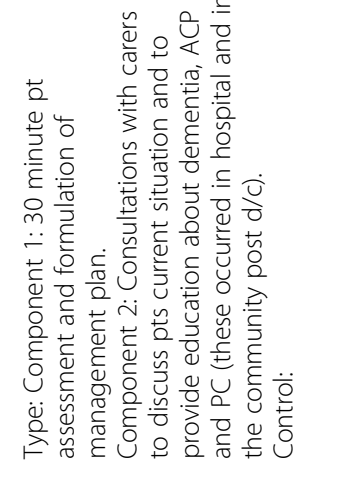 & 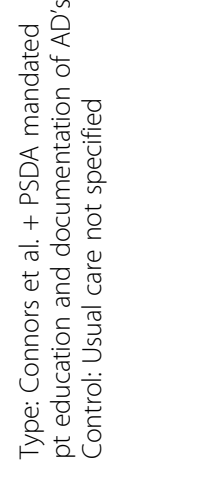 \\
\hline 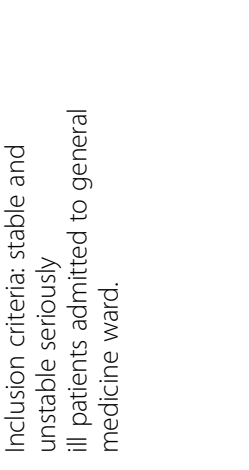 & 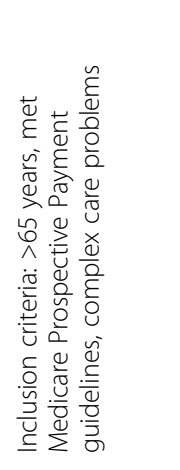 & 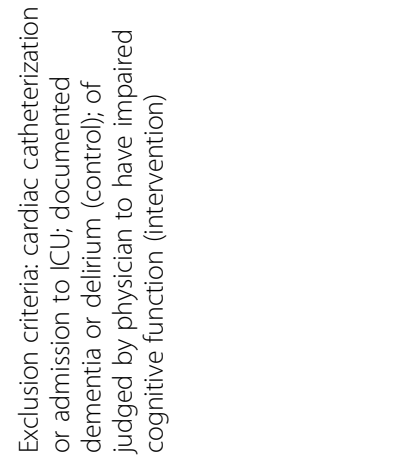 & 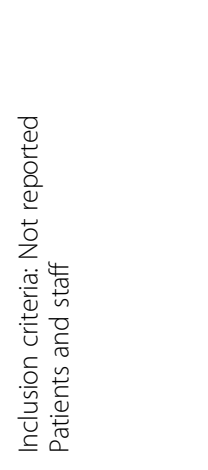 & 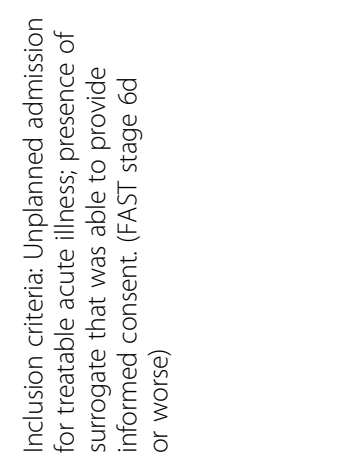 & 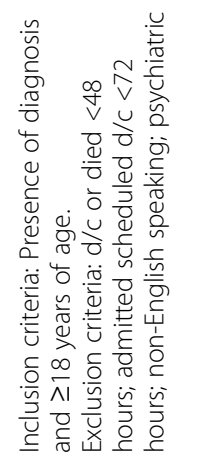 \\
\hline 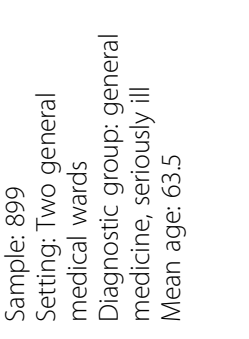 & 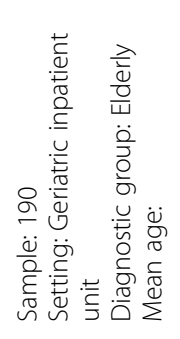 & 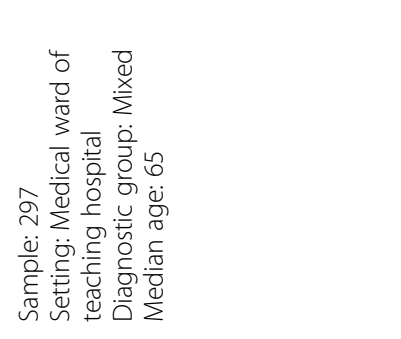 & 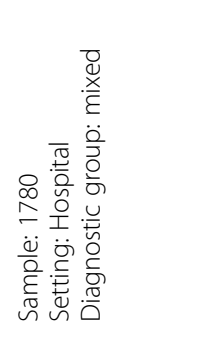 & 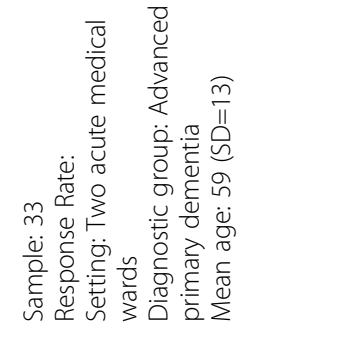 & 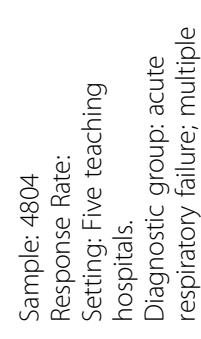 \\
\hline 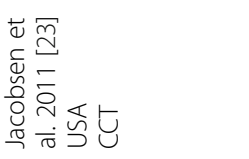 & 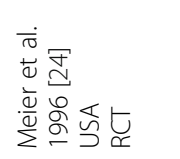 & 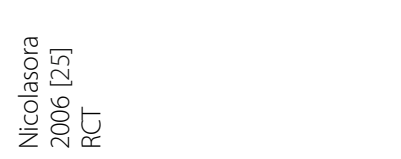 & 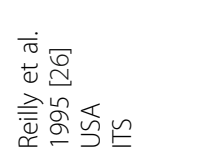 & 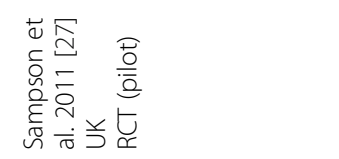 & 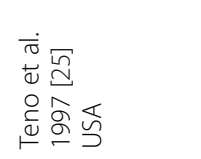 \\
\hline
\end{tabular}



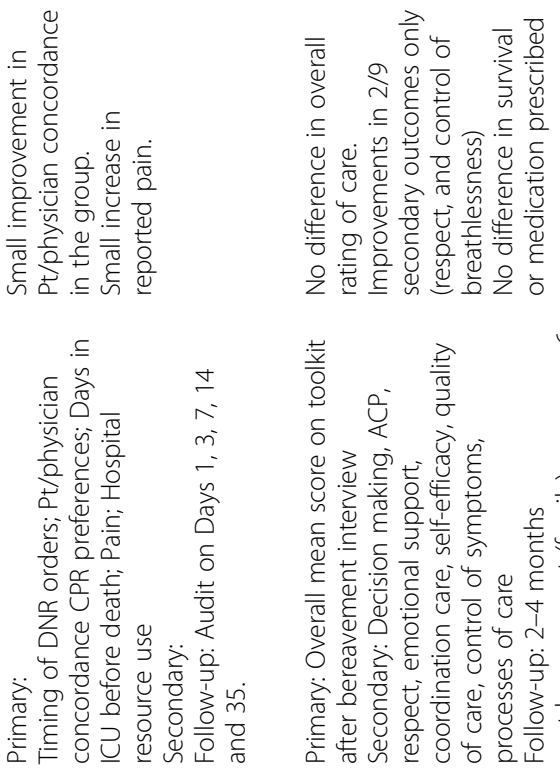

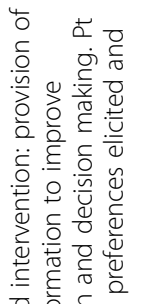

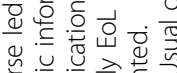

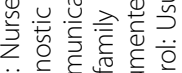

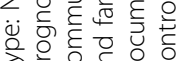

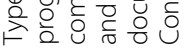

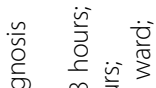

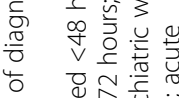

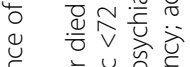

㐫

๗ँ

.ं்

.

突

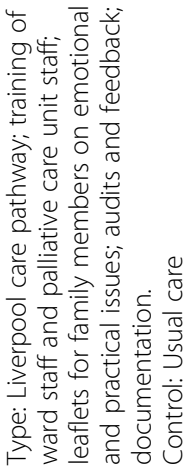

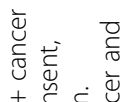

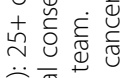

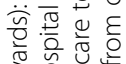

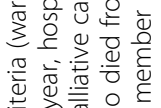

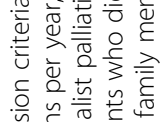

产

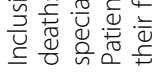

흔.

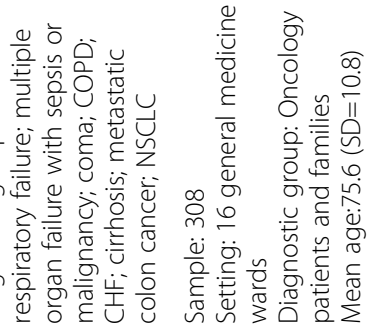
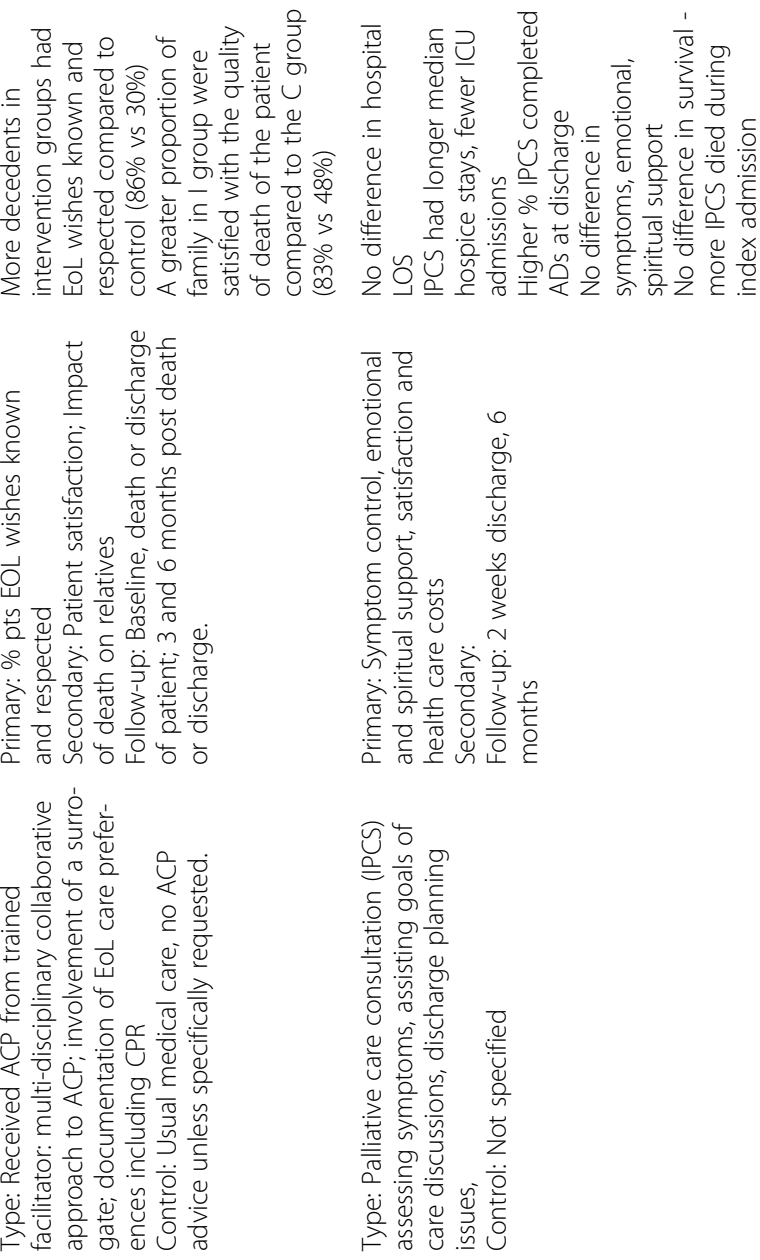

冚

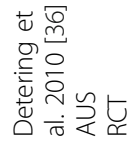

잉 क्ष

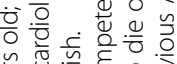

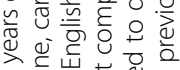

o

i⿱宀

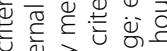

ᄃ

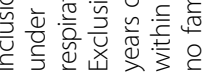

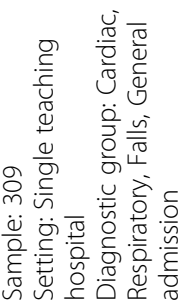

일

능

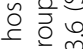

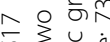

نं

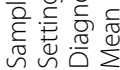

它

용 


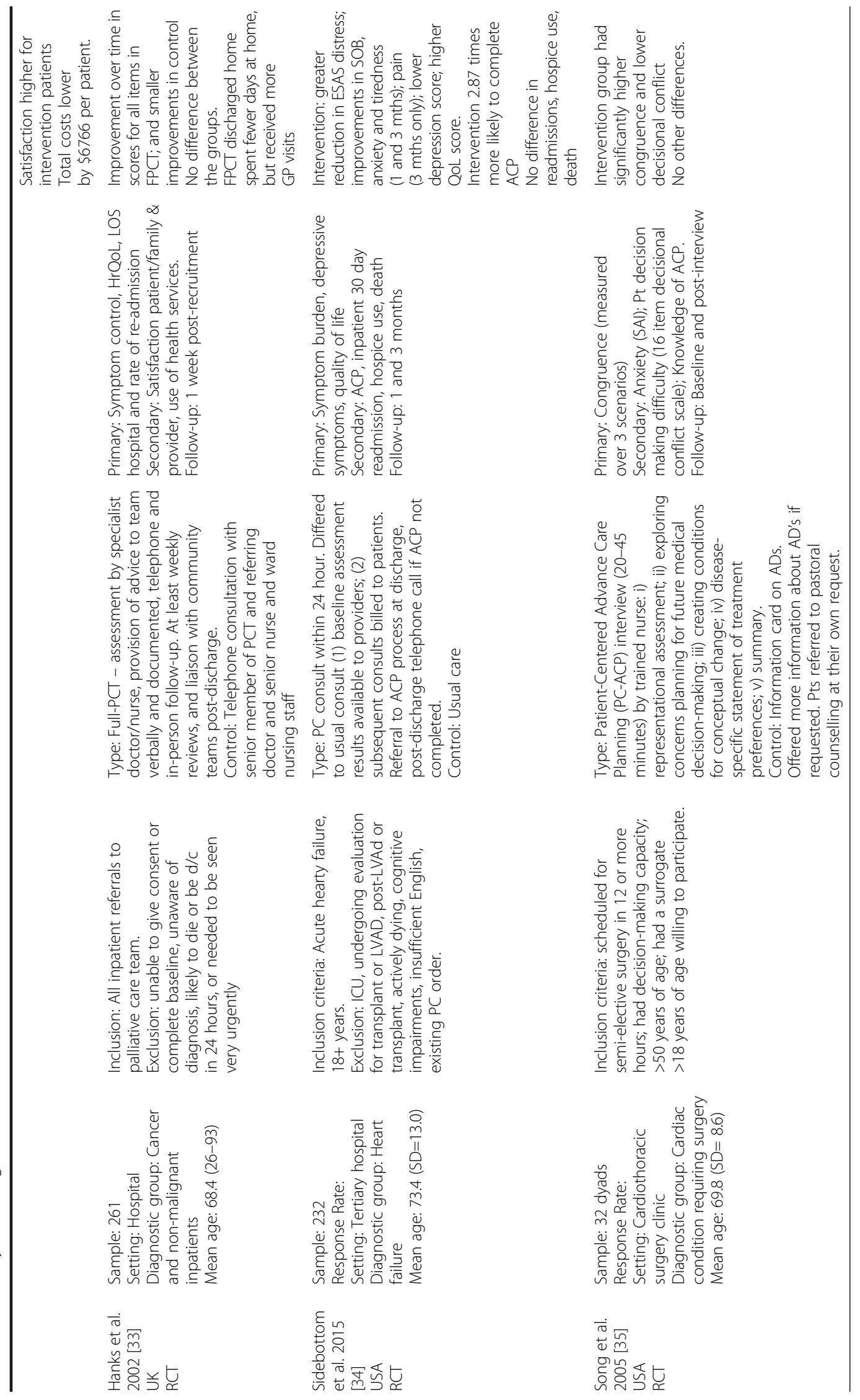




\section{Effectiveness Intervention studies examining end-of-life outcomes}

Two studies examined the impact of facilitated discussions about end-of-life care preferences with patients and support persons. In the SUPPORT trial [30], no significant improvements were found in relation to patient and physician agreement on preferences to withhold resuscitation, pain, hospital resource use or median time until a DNR order was written. However, receiving formal ACP from a trained facilitator improved adherence to wishes; satisfaction, and reduced stress, anxiety, and depression among older inpatients and carers [36]. Three of four studies reported benefits of palliative care consultations on patient outcomes, health care utilisation and costs. Benefits included lower total costs and longer hospice stays [32]; as well as improved symptoms $[33,34]$. No significant difference in carer-perceived overall quality of care was found as a consequence of implementing the Liverpool Care Pathway in 16 Italian hospitals [31].

\section{Discussion}

\section{Volume of research over time and by study type}

The growing number of publications in this field reflects the increasing medical and societal demand for improved end-of-life care in hospitals. Given the methodological problems involved in intervention studies, most published studies are descriptive in nature. Many were comprised of retrospective audits examining receipt of life-sustaining treatments, patient symptoms and end-oflife documentation. Others examined patient and family perceptions of care quality or health status. Few were measurement studies, which may reflect the challenges associated with measuring outcomes of effective end-oflife communication. Only $10 \%$ of the total were intervention studies.

\section{Quality of interventions aimed at improving end-of-life processes and outcomes}

Only 18 of the 48 intervention studies aimed at improving end of life processes met EPOC design criteria. The remainder were historical control trials, which provide potentially promising data on the feasibility and acceptability of different intervention, but require more rigorous testing. Methodological quality of the included intervention studies was variable. Particular attention needs to be paid to reporting on blinding of outcome assessment and methods of generating allocation sequence and concealing allocation.

\section{Effectiveness of interventions examining end of life processes and outcomes}

Patient-directed interventions represent a less resource intensive approach to increasing the uptake of end-of- life processes. However, the potential reach of these interventions may be limited in hospitalised populations. Unstable patients experiencing acute illness and those lacking capacity comprise a significant proportion of hospitalised populations. This group are unlikely to utilise patient-directed interventions. In these cases, the substitute decision maker may be called on to communicate or make decisions on behalf of patients [3], so would make an appropriate alternative target for intervention. Interventions have also typically focused improving certain end-of-life processes, such as completion of ACDs, without acknowledging the potential role that other processes may play [11]. Segmenting care in this manner does not necessarily mirror the patient's experience, nor does it recognise that end-of-life care is often synergistic and may require multiple components to be delivered to achieve a positive impact. For instance, introducing a reminder system to increase rates of endof-life discussions is unlikely to have an impact if patients and staff lack the requisite knowledge and skills to discuss these issues effectively. Hospitals are also made up of individuals with different preferences, skills and motivation to change [6]. Therefore, relying on individuals who are willing and able to be involved in end-of-life research can bias findings. For example, the failure of the landmark SUPPORT trial has been partly attributed to a focus on improving patient-level decision-making without addressing larger, systemrelated challenges [36].

A more efficient and effective approach may be to support the implementation of system-level changes with potential to benefit everyone within the hospital setting. These approaches allow multiple interventions to be delivered in tandem to address deficits across a range of processes and outcomes. However, they can also pose unique challenges in relation to determining which components contribute to positive change [19]. Adopting alternative research designs, such as multiple baseline and stepped wedge designs has the potential to contribute to the evidence while maintaining methodological rigour [37].

Examining the impact of interventions on end-of-life processes alongside outcomes can provide a balanced picture of healthcare delivery, as it can help to determine whether successful implementation of an end-of-life process positively impacts end-of-life outcomes.

However, the extent to which interventions which target end-of-life processes translate to improved end-oflife outcomes is unclear. Mixed benefits of ACP and palliative care interventions were reported in relation to concordance between preferred and actual care, health status, quality of life and health care costs [11]. These findings are consistent with advance care planning reviews of studies undertaken in other care settings [12]. 
Reviews of palliative care interventions in ICU settings suggest that consultative approaches, in which palliative care teams consult with the treating team, may be more effective than approaches which attempt to integrate palliative care principles into the daily routines (i.e. integrative approach) [38]. Further research examining this hypothesis is warranted. Given that these interventions often rely on dedicated resources, evidence of effectiveness and sustainability within variable hospital environments must be established.

\section{Directions for future research}

Strategies that intervene with substitute decision makers as well as patients should be explored, given likelihood of impaired capacity among hospitalised patients [39]. In particular, methodological rigorous studies examining multi-faceted, system-based interventions such as education; checklists or tools; audit and feedback and reminders should be undertaken [39]. Future research efforts should also be focused on evaluating consultative palliative care interventions that aim to ensure patients are getting the right care. Further evidence of the benefits for these more complex interventions on end-of-life outcomes, as well as their sustainability must be established.

Introducing topics such as ACP and palliative care in the community may also help alleviate pressure on hospitals. Currently, this is not done in a systematic way $[40,41]$. Undertaking ACP in the community may allow preferences to be discussed and decisions made outside the context of a health crisis [42]. Increasing awareness about palliative care may lead to more positive impressions, more equitable uptake of services and improved care quality [43]. While ACP uptake is low among the general public, people are willing to discuss their views about end-of-life issues [44]. General practitioners are well placed to engage in advance care planning as they see a significant proportion of the population and will often have contextual knowledge about individuals [45]. However, lack of skills, difficulties with defining the right moment, and fear of depriving patients of hope are often cited as barriers [46]. Strategies that promote interprofessional collaboration between providers in different care settings, including primary care, hospital and residential aged care facilities, are needed [39]. Few such approaches have been rigorously evaluated.

\section{Limitations}

First, the search strategy may have resulted in publication bias, as we did not include non-published studies or grey literature and there is different terminology used in different countries. Second, the authors excluded studies of provider-directed interventions when an assessment of impact on patient outcomes or processes was not included (e.g. studies that examined the impact of communication skills training interventions on provider knowledge alone). While these interactive education approaches are promising; these outcomes were not the focus and have been examined previously.

\section{Conclusions}

There is a lack of methodologically rigorous studies in this field. Publications examining end-of-life care in hospitals are predominately descriptive in nature, with few rigorous trials of interventions aimed at improving the care of the dying. More high-quality intervention trials in hospitals are required to make clear recommendations about which strategies are most effective in improving end-of-life care processes, and whether these improvements translate to improved end-of-life outcomes. Interventions targeting both the patient and their substitute decision maker, and those strategies with the potential to change practice patterns at a system level should be explored.

\section{Additional files}

Additional file 1: Search terms. (DOCX $14 \mathrm{~kb})$

Additional file 2: Summary of outcomes. (DOCX $16 \mathrm{~kb}$ )

Abbreviations

ACD: Advance care directive; ACP: Advance care planning; CPR: Cardiopulmonary resuscitation; DNR: Do-not-resuscitate; EPOC: Effective practice and organisation of care; GOCD: Goals of care discussions; ICU: Intensive care unit

\section{Acknowledgements}

The authors would like to acknowledge the assistance of Angela Smith from Hunter New England Health Libraries who assisted with refining and conducting the literature search.

\section{Funding}

AW is supported by an Australian Research Council Post-Doctoral Discovery Early Career Research Award. ND is supported by a Australian Rotary PhD Scholarship. This research was supported by a Strategic Research Partnership Grant from The Cancer Council NSW to the Newcastle Cancer Control Collaborative.

\section{Availability of data and materials}

This paper is a systematic review. Articles selected for this review were referenced in bibliography. All data extracted from the selected studies were presented in the tables. There is no raw data to be made available.

\section{Authors' contributions}

AW and RSF conceived of and designed the review. ND and AW undertook data extraction. All authors contributed to data analysis, drafting of the manuscript and have read and approved the final manuscript.

\section{Competing interests}

The authors declare that they have no competing interests.

Consent for publication

Not applicable.

Ethics approval and consent to participate Not applicable. 


\section{Publisher's Note}

Springer Nature remains neutral with regard to jurisdictional claims in published maps and institutional affiliations.

\section{Author details \\ 'Priority Research Centre in Health Behaviour, University of Newcastle Callaghan, NSW 2308, Australia. ${ }^{2}$ Hunter Medical Research Institute, Newcastle, NSW 2305, Australia. ${ }^{3}$ University of Sydney, Chris O'Brien Lifehouse, Level 6 North, Missenden Road, Camperdown 2050, Australia. ${ }^{4}$ Faculty of Health and Medicine, University of Newcastle, Callaghan, NSW 2308, Australia. ${ }^{5}$ Hunter New England Local Health District, Newcastle 2305, Australia.}

\section{Received: 13 October 2016 Accepted: 26 April 2017}

Published online: 19 May 2017

\section{References}

1. Australian Institute of Health and Welfare. Lung cancer in Australia: an overview. Cancer series no. 64. Cat. no. CAN 58. Canberra: Australian Institute of Health and Welfare; 2011.

2. National Center for Health Statistics. National Hospital Discharge Survey. 2010

3. Al-Qurainy $R$, Collis $E$, Feuer D. Dying in an acute hospital setting: the challenges and solutions. Int J Clin Pract. 2009;63(3):508-15.

4. World Health Organisation. Global Atlas of Palliative Care at the End of Life. London, UKWorldwide Hospice Palliative Care Alliance; 2014.

5. Lund S, Richardson A, May C. Barriers to advance care planning at the end of life: an explanatory systematic review of implementation studies. PLoS ONE. 2015;10(2):e0116629.

6. Frost DW, Cook DJ, Heyland DK, Fowler RA. Patient and healthcare professional factors influencing end-of-life decision-making during critical illness: a systematic review. Crit Care Med. 2011:39(5):1174-89.

7. Clark K, Byfieldt N, Green M, Saul P, Lack J, Philips JL. Dying in two acute hospitals: would usual care meet Australian national clinical standards? Aust Health Rev. 2014;38:223-229.

8. Wright AA, Keating NL, Balboni TA, Matulonis UA, Block SD, Prigerson HG. Place of death: correlations with quality of life of patients with cancer and predictors of bereaved caregivers' mental health. J Clin Oncol. 2010;28(29): 4457-64.

9. Virdun C, Luckett T, Davidson P, Philips J. Dying in the hospital setting: A systematic review of quantitative studies identifying the elements of endof-life care that patients and their families rank as being most important. Palliat Med. 2015;29(9):774-796.

10. Robinson J, Gott M, Ingleton C. Patient and family experiences of palliative care in hospital: what do we know? An integrative review. Palliat Med. 2014 28:18-33.

11. Houben $\mathrm{CH}$, Spruit MA, Groenen MT, Wouters EF, Janssen DJ. Efficacy of advance care planning: a systematic review and meta-analysis. J Am Med Dir Assoc. 2014:15(7):477-89.

12. Brinkman-Stoppelenburg A, Rietjens JA, van der Heide A. The effects of advance care planning on end-of-life care: a systematic review. Palliat Med. 2014;28(8):1000-25.

13. Khandelwal N, Kross EK, Engelberg RA, Coe NB, Long AC, Curtis JR. Estimating the effect of palliative care interventions and advance care planning on ICU utilization: a systematic review. Crit Care Med. 2015; 43(5):1102-11.

14. Aslakson R, Cheng J, Vollenweider D, Galusca D, Smith TJ, Pronovost PJ. Evidence-based palliative care in the intensive care unit: a systematic review of interventions. J Palliat Med. 2014;17(2):219-35.

15. Swerisson H, Duckett S. Dying Well. Grattan Institute; 2014.

16. Flottorp SA, Oxman AD, Krause J, Musila NR, Wensing M, Godycki-Cwirko M, Baker R, Eccles MP. A checklist for identifying determinants of practice: a systematic review and synthesis of frameworks and taxonomies of factors that prevent or enable improvements in healthcare professional practice. Implement Sci. 2013;8(1):1-11.

17. Grimshaw JM, Eccles MP, Lavis JN, Hill SJ, Squires JE. Knowledge translation of research findings. Implement Sci. 2012;7:50.

18. Group CEPaOoCR. Suggested risk of bias for EPOC reviews. Oslo: Norwegian Knolwedge Centre for Health Services; 2015

19. Bailey FA, Williams BR, Woodby LL, Goode PS, Redden DT, Houston TK, Granstaff US, Johnson 2nd TM, Pennypacker LC, Haddock KS, et al.
Intervention to improve care at life's end in inpatient settings: the BEACON trial. J Gen Intern Med. 2014;29(6):836-43.

20. Cugliari AM, Miller T, Sobal J. Factors promoting completion of advance directives in the hospital. Arch Intern Med. 1995;155(17):1893-8.

21. El-Jawahri A, Mitchell SL, Paasche-Orlow MK, Temel JS, Jackson VA, Rutledge RR, Parikh M, Davis AD, Gillick MR, Barry MJ, et al. A randomized controlled trial of a cpr and intubation video decision support tool for hospitalized patients. J Gen Intern Med. 2015;30(8):1071-80.

22. Grimaldo DA, Wiener-Kronish JP, Jurson T, Shaughnessy TE, Curtis JR, Liu LL. A randomized, controlled trial of advanced care planning discussions during preoperative evaluations. Anesthesiology. 2001;95(1):43-50. discussion 45A.

23. Jacobsen J, Robinson E, Jackson VA, Meigs JB, Billings JA. Development of a cognitive model for advance care planning discussions: results from a quality improvement initiative. J Palliat Med. 2011;14(3):331-6.

24. Meier DE, Fuss BR, O'Rourke D, Baskin SA, Lewis M, Morrison RS. Marked improvement in recognition and completion of health care proxies. a randomized controlled trial of counseling by hospital patient representatives. Arch Intern Med. 1996;156(11):1227-32

25. Nicolasora N, Pannala R, Mountantonakis S, Shanmugam B, DeGirolamo A, Amoateng-Adjepong Y, Manthous CA. If asked, hospitalized patients will choose whether to receive life-sustaining therapies. J Hosp Med. 2006;1(3):161-7.

26. Reilly BM, Wagner M, Magnussen CR, Ross J, Papa L, Ash J. Promoting inpatient directives about life-sustaining treatments in a community hospital. Results of a 3-year time-series intervention trial. Arch Intern Med. 1995;155(21):2317-23.

27. Sampson EL, Jones L, Thune-Boyle ICV, Kukkastenvehmas R, King M, Leurent B, Tookman A, Blanchard MR. Palliative assessment and advance care planning in severe dementia: an exploratory randomized controlled trial of a complex intervention. Palliat Med. 2011;25(3):197-209.

28. Teno JM, Licks S, Lynn J, Wenger N, Connors Jr AF, Phillips RS, O'Connor MA, Murphy DP, Fulkerson WJ, Desbiens N, et al. Do advance directives provide instructions that direct care? SUPPORT investigators. Study to understand prognoses and preferences for outcomes and risks of treatment J Am Geriatr Soc. 1997:45(4):508-12.

29. Ahronheim JC, Morrison RS, Morris J, Baskin S, Meier DE. Palliative care in advanced dementia: a randomized controlled trial and descriptive analysis. J Palliat Med. 2000;3(3):265-73.

30. The SUPPORT Principal Investigators. A controlled trial to improve care for seriously ill hospitalized patients. The study to understand prognoses and preferences for outcomes and risks of treatments (SUPPORT). Jama. 1995; 274(20):1591-1598.

31. Costantini M, Romoli $V$, Leo SD, Beccaro M, Bono L, Pilastri $P$, Miccinesi G, Valenti D, Peruselli C, Bulli F, et al. Liverpool care pathway for patients with cancer in hospital: a cluster randomised trial. Lancet. 2014:383(9913):226-37.

32. Gade G, Venohr I, Conner D, McGrady K, Beane J, Richardson RH, Williams MP, Liberson M, Blum M, Della Penna R. Impact of an inpatient palliative care team: a randomized control trial. J Palliat Med. 2008;11(2):180-90.

33. Hanks GW, Robbins M, Sharp D, Forbes K, Done K, Peters TJ, Morgan H, Sykes J, Baxter K, Corfe F, et al. The imPaCT study: a randomised controlled trial to evaluate a hospital palliative care team. Br J Cancer. 2002;87(7):733-9.

34. Sidebottom AC, Jorgenson A, Richards H, Kirven J, Sillah A. Inpatient palliative care for patients with acute heart failure: outcomes from a randomized trial. J Palliat Med. 2015;18(2):134-42.

35. Song M-K, Kirchhoff KT, Douglas J, Ward S, Hammes B. A randomized, controlled trial to improve advance care planning among patients undergoing cardiac surgery. Med Care. 2005;43(10):1049-53.

36. Detering KM, Hancock AD, Reade MC, Silvester W. The impact of advance care planning on end of life care in elderly patients: randomised controlled trial. BMJ. 2010;340:C1345.

37. Sanson-Fisher RW, D'Este CA, Carey ML, Noble N, Paul CL. Evaluation of systems-oriented public health interventions: alternative research designs. Annu Rev Public Health. 2014;35:9-27.

38. Fawole OA, Dy SM, Wilson RF, Lau BD, Martinez KA, Apostol CC, Vollenweider D, Bass EB, Aslakson RA. A systematic review of communication quality improvement interventions for patients with advanced and serious illness. J Gen Intern Med. 2013;28(4):570-7.

39. RozedesOrdons AL, Sharma N, Heyland DK, You JJ. Strategies for effective goals of care discussions and decision-making: perspectives from a multicentre survey of Canadian hospital-based healthcare providers. BMC Palliat Care. 2015;14(1):1-10. 
40. Glaudemans JJ, Moll van Charante EP, Willems DL. Advance care planning in primary care, only for severely ill patients? A structured review. Fam Pract. 2015;32(1):16-26.

41. Sellars M, Detering KM, Silvester W. Current advance care planning practice in the Australian community: an online survey of home care package case managers and service managers. BMC Palliat Care. 2015;14:15.

42. Scott IA, Mitchell GK, Reymond EJ, Daly MP. Difficult but necessary conversations-the case for advance care planning. Med J Aust. 2013; 199(10):662-6

43. Mcllfatrick S, Hasson F, McLaughlin D, Johnston G, Roulston A, Rutherford L, Noble H, Kelly S, Craig A, Kernohan WG. Public awareness and attitudes toward palliative care in Northern Ireland. BMC Palliat Care. 2013;12(1):1-7.

44. Goodridge D, Quinlan E, Venne R, Hunter P, Surtees D. Planning for serious illness by the general public: a population-based survey. ISRN Family Med. 2013;2013:483673.

45. De Vleminck A, Pardon K, Beernaert K, Houttekier D, Vander Stichele R, Deliens L. How do general practitioners conceptualise advance care planning in their practice? A Qualitative Study. PLoS ONE. 2016;11(4): e0153747.

46. De Vleminck A, Houttekier D, Pardon K, Deschepper R, Van Audenhove C, Vander Stichele R, Deliens L. Barriers and facilitators for general practitioners to engage in advance care planning: a systematic review. Scand J Prim Health Care. 2013;31(4):215-26

\section{Submit your next manuscript to BioMed Central and we will help you at every step:}

- We accept pre-submission inquiries

- Our selector tool helps you to find the most relevant journal

- We provide round the clock customer support

- Convenient online submission

- Thorough peer review

- Inclusion in PubMed and all major indexing services

- Maximum visibility for your research

Submit your manuscript at www.biomedcentral.com/submit 